Horizons philosophiques

\title{
Démocratie et expérience : introduction à la démocratie créatrice de John Dewey
}

\section{François Leroux}

Volume 5, numéro 2, printemps 1995

Le philosophe et l'état

URI : https://id.erudit.org/iderudit/800978ar

DOI : https://doi.org/10.7202/800978ar

Aller au sommaire du numéro

Éditeur(s)

Collège Édouard-Montpetit

ISSN

1181-9227 (imprimé)

1920-2954 (numérique)

Découvrir la revue

Citer cet article

Leroux, F. (1995). Démocratie et expérience : introduction à la démocratie créatrice de John Dewey. Horizons philosophiques, 5(2), 20-40.

https://doi.org/10.7202/800978ar d'utilisation que vous pouvez consulter en ligne.

https://apropos.erudit.org/fr/usagers/politique-dutilisation/ 


\section{DÉMOCRATIE ET EXPÉRIENCE : INTRODUCTION À LA DÉMOCRATIE CRÉATRICE DE JOHN DEWEY}

L'essai que l'on va lire estle texte d'une conférence préparée en 1939 par John Dewey à l'occasion d'un congrès organisé en l'honneur de ses 80 ans. II s'en faudrait de beaucoup qu'on y retrouve l'ensemble des préoccupations de l'auteur à propos de la démocratie : en tracer le tableau nécessiterait un travail tout autre que celui-ci'. Mais le petit essai que nous présentons indique avec clarté certains des aspects les plus importants de la pensée politique du philosophe. Le nom de John Dewey reste, on le sait, à jamais associé à l'engagement social et théorique que le problème de la démocratie a suscité chez lui et qui a gouverné l'essentiel de ses interventions et de son œuvre ${ }^{2}$. C'est un lieu commun du commentaire de présenter Dewey comme le philosophe de la démocratie américaine, comme le penseur qui “...est l'incarnation de l'Amérique, de sa conscience la plus sensible, de son intelligence constructive et de son intense foi démocratique ${ }^{3}$ ". Si les lignes écrites par Dewey en 1939 dont nous procurons ici la traduction ne démentent pas entièrement ces affirmations, c'est que ces dernières ne sont pas sans raisons : elles sont loin, toutefois, de

1. L'intérêt de Dewey pour la démocratie peut-être retracé très tôt dans l'œuvre : on pense ici au long essai de 1888 intitulé "The Ethics of Democracy" consacré à la critique la plus sévère de la charge menée par Sir Henry Maine contre la démocratie dans son ouvrage de 1885, Popular Government.

2. Le lecteur désireux de prendre connaissance du parcours social et intellectuel de Dewey et de mieux connaître la place que l'interrogation sur la démocratie y occupe pourra consulter notamment l'importante recherche d'ensemble menée par Robert B. Westbrook dans son ouvrage John Dewey and American Democracy (Cornell University Press, Ithaca and London, 1991) ainsi que l'article de Richard J. Bernstein, "Dewey et la démocratie : la tâche qui nous attend", in La pensée américaine contemporaine, sous la direction de John Rajchman et Cornel West, Paris, P.U.F., coll. «Philosophie d'aujourd'hui», 1991, p. 119-121.

3. S. Ratner in The Philosophy of the Common Man - Essays in Honor of John Dewey, cité par Gérard Deledalle, La Philosophie Américaine, Bruxelles, De BoeckWesmael, 1987, p. 169. 
révéler toute la ressource du texte.

Une lecture de La démocratie créatrice : la tâche qui nous attend pourrait, en effet, s'organiser à partir du triple renvoi soigneusement marqué par Dewey dans sa conférence : renvoi à l'origine d'abord, à l'actualité ensuite et à l'action, finalement. On retrouverait là, d'ailleurs, le dispositif ayant donné forme à l'image que l'on se fait le plus souvent du penseur et de son œuvre : image de Dewey présenté comme penseur "yankee", comme penseur libéral et comme penseur pragmatiste. Les indications ici ne manquent évidemment pas, ni les difficultés qu'elles nourrissent très souvent. Ainsi, comment ne pas remarquer que l'auteur de La démocratie créatrice... inscrit dès l'entrée la référence à sa nationalité, plus précisément au programme politique porté par la République américaine depuis la conquête de son indépendance, soit "la création de la démocratie"? Plus encore:en passant sous silence les exactions allant jusqu'au projet de génocide dont les peuples autochtones ont été victimes en sol américain comme ailleurs et auxquels les fondateurs de son pays, ces «hommes les plus expérimentés et les plus sages" dontil parle, doivent être aussi associés, Dewey ne cède-t-il pas d'emblée à un des mythes politiques les plus puissants de sa "nation»? S'il s'agit là d'une des limites les plus sévères de son analyse, il reste que ce destin ayant présidé à la naissance du pays, et vu comme plutôt favorable par le penseur, ne devait pas faire oublier totalement quelques unes des crises dont elle constituait le dénouement provisoire: en rappelant son âge, le philosophe ne pouvait manquer de situer l'invention de la démocratie américaine dans le contexte de quelques unes des guerres internes et externes les plus importantes qu'elle aura dû affronter. Ce n'est évidemment pas là le seul repère fourni par La démocratie créatrice...: mais audelà des réserves qu'appelle le silence du penseur sur certains épisodes de l'histoire américaine, il faut noter que l'urgence de poser à nouveau la question de la démocratie se révèle, dans l'essai de 1939, comme intimement relié à la fois aux défis sociaux immenses que le pays devait affronter, comme aux événements ayant déjà commencé à engager tout un peuple 
dans un conflit qui n'allait pas s'éteindre avec la chute de l'Allemagne hitlérienne. Nous aurons à y revenir.

Interroger la démocratie n'aura donc pu se faire, pour Dewey, qu'en se rapportant aux crises ayant présidé à sa naissance comme à celles qui, déjà, la forçaient à se renouveler. L'évocation du territoire et de sa conquête déposée dès les premiers moments du texte est, à cet égard, décisive. La crise qui affecte un peuple s'assimile ici, il est vrai, à une limite à franchir et à un espace à redéfinir : aussi typiquement "américaine" qu'elle puisse apparaître, cette métaphorique décrit un rapport complexe. D'une part, le philosophe, tout en rappelant certaines des décisions politiques prises dès les premiers moments de la démocratie américaine, veut établir que ce rapport à l'origine ne ramène pas simplement aux commencements. En ce sens, la lecture peut aisément se laisser conduire par une certaine linéarité de la métaphore: Dewey ne paraît-il pas inviter, dans La démocratie créatrice..., à comprendre qu'après avoir été, pour l'Amérique, continentale, la «frontière" devint par la suite sociale et qu'elle devaitêtre repérée désormais à l'échelle mondiale? Mais si ce parcours correspond à un scénario observable, il ne doit pas nous faire rater le mouvement du texte définissant le territoire à "conquérir» comme essentiellement «moral» et situant le débat sur la démocratie en terme de frontière(s) ouverte(s) sur le politique, c'est-à-dire en terme d'aménagement de ce que Dewey appellera dans sa conférence "un lieu de vie en commun». L'enjeu, ici, n'est évidemment pas spécifiquement "américain». D'autre part, la démocratie désigne pour le philosophe une forme particulière du temps historique, un mode différencié de la conscience historique : ce n'est pas l'événement ou la structure qui doivent à eux seuls modeler le rapport politique, mais ce que le penseur désigne par le terme d'expérience. La signification à accorder à cette idée exigera plus d'explications; disons simplement, pour l'instant, que chez Dewey, la démocratie introduit une expérience autre de l'autorité et doit consacrer d'abord et avant tout l'autorité même de l'expérience. 
II ne suffirait donc pas de dire que le texte de La démocratie créatrice... est un texte engagé parce qu'il inscrit clairement la référence à l'histoire (nationale) américaine et invite à penser la démocratie en opposition aux totalitarismes qui en constituaient alors la menace extérieure la plus immédiate. Dans son essai, Dewey ne manque pas de dire que c'est comme philosophe qu'il intervient. Ce cadrage, avant tout renvoi à l'histoire ou aux institutions, en fait d'emblée un texte politique. Est-il, en effet, besoin de rappeler longuement que l'aventure intellectuelle et sociale du penseur a constitué au premier chef un intense plaidoyer en faveur d'une réassignation de la philosophie, un travail littéralement militant pour son installation résolue dans l'espace public où elle doit participer sans réserves aux débats du jour et apporter toute la clarification possible aux questions les plus immédiates comme les plus techniques se posant aux membres de la Cité ${ }^{4}$ ? C'est dire que tout texte philosophique pour Dewey doit être un texte de combat : et le combat de la philosophie doit au premier chef être le combat pour la démocratie.

La vie et l'œuvre de John Dewey reflètent pleinement cette conviction. John Dewey est né à Burlington, dans le Vermont, le 20 octobre 1859, quelques heures après l'emprisonnement de l'abolitionniste John Brown à la suite du raid qu'il mena contre l'arsenal de Harper's Ferry en Virginie. Dewey est mort à New-York le 1er juin 1952 alors que la guerre de Corée faisait rage. Les biographes ne manquent pas de signaler que la famille de Dewey, installée aux Etats-Unis depuis plusieurs générations, participa activement à la formation de l'Union. Après ses études secondaires, Dewey s'inscrivit à l'Université du Vermont. II enseigna par la suite dans un High School de

4. C'est en 1917 que Dewey donnera une expression serrée et rigoureuse de ce point de vue dans "The Need for a Recovery of Philosophy». D'une façon générale, le lecteur qui chercherait à connaître les fondements et les prolongements de cette prise de position devrait prendre connalssance du célèbre ouvrage de Dewey Reconstruction in Philosophy (Enlarged Edition, with a New Introduction by the Author), Beacon Press, Boston, 1957. 
Pennsylvanie et dans la région de Burlington. Encouragé par un de ses anciens professeurs H.A.P. Torrey et sur les conseils de W.T. Harris, directeur du Journal of Speculative Philosophy dans lequel il avait fait paraître un premier article, Dewey se rendit en 1882 à l'université John Hopkins, à Baltimore, pour y poursuivre des études en philosophie. C'est G.S. Morris qui alors l'introduisit à la pensée hégélienne et ce fut G.S. Hall qui l'initia à la psychologie expérimentale. Dewey ne fut pas attiré par le grand pragmatiste C.S. Pierce qui enseignait alors à John Hopkins comme professeur invité. C'est une thèse sur la psychologie de Kant qui lui mérita son doctorat en 1884. Appelé à diriger le département de philosophie à l'université du Michigan, Morris invita Dewey à le joindre. Il y enseigna dix ans avec une interruption d'un an, en 1888, à l'université du Minnesota. Durant cette année, Morris mourut et ce fut Dewey qui fut appelé à lui succéder à Ann Arbor.

C'est à cette époque que l'accent mis sur la psychologie dans les recherches faites par Dewey jusqu'alors se déplaca au profit de la réflexion éthique et politique. On attribue ce changement à la lecture par Dewey du penseur hégélien et libéral T.H. Greene ainsi qu'à son mariage en 1886 avec Alice Chipman. Etudiante de Dewey à l'université du Michigan où elle suivit quelques uns de ses séminaires avancés, Alice Chipman intéressa Dewey par ses idées libérales et l'invita à mesurer la misère et les tensions sociales engendrées par l'expansion du capitalisme américain. Dans son étude sur John Dewey ${ }^{5}$, Cornel West rappelle quelques aspects généraux de cette crise interne des Etats-Unis d'Amérique dont l'aggravation tout au long de la première moitié du vingtième siècle constituera pour l'auteur de La démocratie créatrice... le premier motif de son intervention politique. Très tôt, en effet, dans son œuvre, Dewey se convainc que la démocratie risque de perdre sa signification aux yeux mêmes de ceux qui doivent la faire vivre et qui sont aux prises avec des défis sociaux considérables. Entre 1860 et 1900, la population des États-Unis passe de 31 millions

5. The American Evasion of Philosophy - A Genealogy of Pragmatism, The University of Wisconsin Press, Madison, Wisconsin, 1989, p. 69-111. 
d'habitants à 76 millions, dont 14 millions d'immigrants. Le pays connaît une longue phase de développement économique compliquée par des ralentissements, des périodes de stagnation, etc. Le secteur industriel et manufacturier s'impose dans une économie où régnaitjusqu'alors la production agraire qui restera parailleurs importante. Ce déplacement de l'activité économique rend possible le développement d'une classe ouvrière nombreuse et d'une nouvelle bourgeoisie (petite et grande et, dans ce dernier cas, véritablement oligarchique). Aux effets massifs de cette restructuration du Capital s'ajoute une crise sociale et urbaine majeure : et, dans ce contexte, le besoin d'ajuster les pratiques culturelles - dont les pratiques éducatives, qui retiendront tant Dewey - aux transformations en cours se fait de plus en plus pressant. Dewey analyse l'important bouleversement affectant le tissu social de l'Amérique comme touchant la multiplicité des individus dont les capacités de travail et de développement personnel se trouvent largement ruinées faute d'avoir la chance et les moyens de se déployer, alors que les milieux de vie traditionnels sont abandonnés ou privés des ressources qui permettaient d'en faire jusqu'alors de véritables communautés.

À plus de quarante ans de distance, la conférence de Dewey sur La démocratie créatrice... retraduira la profonde préoccupation du penseur pour ces graves divisions sociales qui menacent la vie même de la démocratie. Le troisième paragraphe de l'essai de 1939 se lit comme suit :

De nos jours, le territoire à conquérir n'est pas physique, mais moral. L'époque des terres vierges qui semblaient s'étendre à l'infini est révolue. Les ressources utilisées sont humaines plutôt que matérielles. Les terres en friche sont les hommes et les femmes adultes qui n'ont pas de travail, les hommes et les femmes jeunes qui se butent à des portes fermées là où, autrefois, ils auraient pu tenter leur chance. La crise qui, il y a cent cinquante ans, réclamait de l'inventivité en matière sociale et politique, nous la vivons aujourd'hui sous une forme qui exige plus de créativité ${ }^{6}$.

6. Nous utilisons ici la traduction de Sylvie Chaput présentée dans ce numéro. 
Plusieurs commentateurs ont insisté sur le profil idéologique et politique de la créativité dont parle le philosophe et que la conférence de 1939 paraît accréditer de ses formulations : celui du libéralisme. Là encore, les choses sont moins simples qu'elles en ont l'air. Bien qu'il ait pu au cours de sa carrière se réclamer d'un «libéralisme radical», le terme ne va pas sans difficulté pour décrire les positions du penseur. Les critiques faites par Dewey des perspectives du libéralisme économique classique sont nombreuses et, rejetant le "laissez-faire" de plusieurs de ses contemporains, il croyait nécessaire l'intervention de l'État dans la régulation des processus de changement affectant la société dans son ensemble.

On pourrait se croire mieux servi, pour identifier Dewey à toute une tradition politique, en évoquantl'individualisme auquel sa pensée semble donner expression. Ainsi, l'essai de 1939 souligne bien l'idée que la démocratie doit être comprise comme une manière personnelle de vivre, qu'elle signifie avoir et manifester constamment certaines attitudes qui forment le caractère individuel et qui déterminent le désir et les fins dans toutes les relations de l'existence ${ }^{7}$ "; de surcroît, Dewey insiste sur une notion de l'égalité et de la liberté qui rejoint celle des libéraux les mieux connus :

La notion démocratique de l'autorité est généreuse. Elle s'applique à tous. Elle est la conviction que chacun est capable de mener sa propre vie sans avoir à subir de contraintes nià recevoir de commandements de quiconque, du moment que sont mises en place les conditions nécessaires ${ }^{8}$.

Mais cet "homme du commun" dont parle le philosophe n'est pas une simple individualité au sens où celle-ci serait la marque d'une égalité formelle ou, nous l'évoquions plus haut, d'une simple identité sociale (nationale). Dewey est intimement persuadé que l'idée de démocratie est la seule qui donne accès

7. Dewey, J., La démocratie créatrice..., sixième alinéa.

8. Dewey, J., La démocratie créatrice..., huitième alinéa. 
- en terme de moyens comme en terme de fins - au plein potentiel de chacun et que la mise en rapport des singularités les mieux affirmées peut seule donner vie à des communautés qui rassemblent véritablement les hommes. La démocratie n'est pas pour Dewey le temps du nombre, et si l' "homme du commun" est un héros apparemment sans nom, il n'est pas sans génie ni visage. Voilà ce qu'il faut penser et que l'essai de 1939 arrive à peine à effleurer : la démocratie ne peut être que confrontation des finitudes et c'est en permettant à la singularité de chacun de se définir que, du fond de ce que l'on pourrait bien appeler des solitudes, s'ouvre un accès au politique ou encore, dans les termes mêmes de La démocratie créatrice..., à la communication. Ce terme nous retiendra bientôt.

Mais l'image de Dewey présenté comme penseur, sinon comme idéologue du libéralisme se nourrit non moins abondamment de son idéalisme politique. Passons rapidement sur le positivisme apparent des dernières lignes de La démocratie créatrice... et sur le moralisme - avec toute l'insistance mise à parler de la démocratie à partir de la quotidienneté - dont Dewey se révèle capable : ils en font sourire plusieurs, de manière plus discrète il est vrai quand ces traits de la pensée du philosophe invitent à réfléchir sur les liens entre démocratie et éducation. On risque d'avoir moins de réserve quand l'appel à la tolérance de l'essai de 1939 paraît adopter une tournure irénique. Ce n'est pas le refus de "la violence» affirmé par Dewey qui peut exaspérer : il est, en effet, difficile de s'opposer à ce propos voulant que la démocratie doive fonder le débat public sur la persuasion et qu'il est impossible de confondre cette dynamique avec celle des totalitarismes où s'exercent les formes les plus violentes du contrôle social et de l'intimidation. Ce qui risque bien d'apparaître comme incongru, c'est cette idée qu'ultimement la solidarité possible en démocratie puisse être assimilée à de l'amitié! Plus encore : quelle valeur accorder à cette perspective qui minimise jusqu'à le faire disparaître à peu près le rôle des conflits entre groupes d'intérêts et classes dans la dynamique des transformations sociales et, postulant que tous peuvent faire preuve de sens commun, c'est-à-dire 
assumer sans défaillir leur tâche de citoyen, fait reposer la défense de la démocratie sur l'effort individuel ${ }^{9}$ ? Seul le renvoi opéré par Dewey dans sa conférence à l'actualité , aux événements les plus importants du temps peut tempérer l'ardeur de la critique. Car le philosophe, loin d'ignorer la dimension du confliten matière de politique, cherchea l'inscrire différemment. On sait, en effet, qu'au moment où il écrit son essai, le penseur en est arrivé à la conclusion qu'il faut cesser de confier à l'institution le soin de fonder et de garantir la vie de la démocratie : et c'est la menace posée par l'action des États totalitaires qui l'en a convaincu.

C'est donc à partir de ces prémisses que le philosophe axera son intervention pour proposer à ses concitoyens comme principale finalité politique le développement intellectuel et moral de chaque membre de la Cité : en d'autres termes, il s'agit de travailler à l'organisation d'une vie sociale qui permette à tous et à chacun d'exercer sa plus large autonomie possible dans une communauté suffisamment forte pour limiter l'intervention de l'État. Cette "démocratie créatrice», Dewey va militer pour sa mise en œuvre par une série d'engagements plus directement politiques dont nous reparlerons. A un niveau disons plus social, il va souhaiter que cette démocratie pluraliste et libérale à laquelle il songe se fonde surl'action d'un processus éducatif imprégné de l'esprit scientifique, c'est-à-dire par cette démarche qui valorise l'enquête, l'expérimentation, la transformation de la théorie et des idées au contact des faits nouveaux. On reconnaîtra là le modèle philosophique du

9. Dans son livre Authority and the Liberal Tradition - From Hobbes to Rorty (Transaction Publishers, New Brunswick (U.S.A.) and London, England, 1992), Robert Heineman retraduit à sa manière cette critique : "For all of his discussion about utilisation of scientifically based philosophical approches in government, Dewey's view of the citizen who would participate in such government was derived from what can only be described as a tremendous leap of faith on his part. His vision of the democratic citizen was thoroughly optimistic and egalitarian, characteristics that seemed to be inherent in his philosophical position. (...) In his discussions of democratic government, Dewey appears to have been thinking in terms of a reconstituted citizen, one who could be trusted to make sound judgements and to act unselfishly, for he had reservations about the citizens of his day". p. 121-122. 
pragmatisme dontil sera bientôt question: c'est cette perspective qui permet au penseur d'articuler les finalités politiques qu'il met de l'avant aux moyens de leur mise en œuvre; et l'on verra que pour Dewey il n'aura jamais été question d'énoncer quelque fin que ce soit en des termes autres que ceux qui permettent de fixer des moyens.

À l'initiative de son ami et collègue James $H$. Tufts, Dewey accepta en 1894 un posteà la John D. Rockefeller University de Chicago. II y fonda la célèbre école expérimentale qui allait porter son nom. Nouvelle expression du besoin d'action de Dewey, son "École-laboratoire» se présentait comme une intervention concrète dans la mise en place d'un système d'éducation totalement renouvelé, proposant à la fois les éléments de culture générale requis pour la démocratie américaine et les éléments plus "fonctionnels" d'éducation permettant la meilleure insertion possible dans un marché du travail en pleine évolution. L'expérience de l' «École-laboratoire» allait prendre fin en 1904 avec la démission de Dewey de l'université de Chicago : les manœuvres du recteur William R. Harper ayant conduità l'intégration de l'École à l'université sans l'accord de Dewey ni des parents des élèves, le philosophe prit congé définitivement et voyagea pendant quelque temps en Europe. La nouvelle de sa démission poussa l'université Columbia, à New York, à créer le poste qui allait lui permettre d'enseigner de 1905 jusqu'à 1930, date où il prit sa retraite. Comme l'écrit Cornel Wes : «C'est au Michigan que Dewey put entreprendre sa carrière, et c'est à Chicago qu'il excella, mais à New York il devint un géant ${ }^{10}$ ". L'œuvre du penseur à Columbia connut alors son véritable tournant pragmatique : ses écrits logiques et ses textes sur l'expérience produits durant cette période le traduisent pleinement ${ }^{11}$. Arrêtons-nous rapidement sur cet aspect de l'œuvre.

10. West, C., The American Evasion of Philosophy..., p. 85 (nous traduisons).

11. On pense icl aux Essays in Experimental Logic (1916), à Experience and Nature (1925) et The Quest for Certainty (1929). A cette liste on ajoutera le grand livre écrit apress la retraite : Logic - The Theory of Inquiry (1938). 
Ce paradigme philosophique qu'est le pragmatisme avait pris forme précise, dans le cas de Dewey, à Chicago où il en avait élaboré avec ses collègues cette version que l'on appelle l'«instrumentalisme". Les historiens de la philosophie nous rappellent, en effet, que le mouvement de pensée que nous connaissons sous le nom de pragmatisme n'a pas une origine simple et qu'il a sa source tant chez C.S. Pierce et William James et les réunions informelles qu'avec d'autres chercheurs ils tinrent à Cambridge, Massachusetts, que chez Dewey et ses collègues de la fameuse Ecole de Chicago. Sans chercher à réduire le pragmatisme en en faisant essentiellement une théorie particulière de la vérité, il n'est pas impossible pour le décrire brièvement d'emprunter cette direction. On peut dire du pragmatisme qu'il permet «...de définir la nature des idées en termes d'opérations à réaliser et d'éprouver la validité des idées par les conséquences de ces opérations ${ }^{12}$ ". Une autre formulation brève retraduit ce point de vue; pour un pragmatiste :

"Le fait est l'idée que rien ne contredit». L'idée est un «fait possible» maintenu dans l'esprit à titre d'hypothèse. Le processus par lequel le fait possible ou idée devient réel est la vérification.(...) II n'y a pas d'autre preuve pour une idée que celle-ci: "La capacité qu'elle a de marcher, d'organiser les faits» ${ }^{13}$.

Cette théorie de la vérité mise de l'avant par le pragmatisme n'est pas que logique : elle est aussi éthique. Dans cette perspective, le Vrai est un aspect du Bien pour autant que l'on se rappelle que l'accès au Vrai exige que les conditions de possibilité d'une vérification authentique de l'idée soient réunies et que les procédés d'enquête sur l'efficacité et la capacité de l'idée d'organiser les faits soient disponibles. C'est donc l'existence du Bien qui rend possible le déploiement du Vrai.

Pour le pragmatiste qu'est Dewey, les idées sont donc des moyens pourl'action: pour s'assurer de leur développement comme pour pouvoir (ré)organiser avec les meilleures chances

12. Dewey, J., cité par Gérard Deledalle, La philosophie Américaine, p. 54.

13. Deledalle, G., La Philosophie Américaine, p. 67. 
de succès le champ des pratiques, il faut donc concevoir et formuler les idées de manière à ce qu'elles exhibent les «instruments" ou les possibilités de transformation qu'elles procurent. Si découvrir la signification des idées c'esten chercher les conséquences, alors il n'y a de vérité que publique et l'on ne s'étonnera pas que Pierce ait pu parler, de son côté, de la "notion démocratique de la vérité». Pour Dewey, le problème de la vérité sera donc aussi un problème social et l'on saisit par là le lien intime entre les préoccupations "logiques" ou "épistémologiques» du philosophe et son énergique défense de la démocratie conçue comme cadre social et politique permettant la libre expression et la libre circulation des idées : à ce titre, elle vaut pour notre penseur comme condition préalable générale de toute vérité et de tout progrès. Cela signifie aussi qu'il faut renoncer, sous peine de subordonner entièrement la vie politique d'un peuple à l'action de l'État, à voir la démocratie comme une modalité extérieure essentiellement délimitée par sa réalité institutionnelle. Pour Dewey, la démocratie est donc une liberté à concevoir si radicalement qu'il en fait la condition même de toute expérience, si l'on veut bien entendre ici par ce mot la qualité créatrice et auto-régulante que l'action individuelle et collective peut exhiber quand elle n'est pas entravée. L'individualismeapparent du propos du philosophe ne doit pas tromper : c'est, d'une certaine manière, un véritable ethos que doit constituer l'idée de démocratie si la liberté qu'elle délimite doit être effective pour chacun.

C'est dire que pour Dewey, la démocratie ne peut être ramenée à son concept : elle est un processus qu'il décrit comme "éducatif» et qui permet d'envisager la vie politique dans une perspective de "communication". Or ce dernier terme, s'il désigne, en un premier sens, les conditions matérielles nécessaires à la mise en œuvre même d'un débat public permettant de renforcer le lien social, définit du même coup I'horizon normatif de ce débat. Les derniers moments de l'essai de 1939 paraissent confier exclusivement à la science le soin de traduire cet idéal : il ne nous paraît pas cependant que le pragmatisme politique de Dewey se ramène à un positivisme. 
En effet, La démocratie créatrice... ne nous présente pas l'activité de la science comme appréhension de ce qui est mais comme ouverture sur ce qui peut être; plus précisément, l'idéal de connaissance que le philosophe souhaiterait voir animer intensément la vie politique, permettrait de détacher de manière radicale l'autorité de ses représentations : «La démocratie est la conviction que le processus de l'expérience importe davantage que tel ou tel résultat particulier - les résultats particuliers ayant une valeur ultime uniquement s'ils servent à enrichir et à ordonner la suite du processus ${ }^{14}$ ".

On sait que certains héritiers de la pensée de Dewey jugeront qu'une telle conception de l'expérience invite à rejeter toute tentative pour définir un fondement au politique ou, comme le dira Richard Rorty, à consacrer «le primat de la démocratie sur la philosophie ${ }^{15}$ ". II s'agit là d'un vaste débat qu'on ne saurait aborder pour lui-même ici. II n'est pas du tout sûr, toutefois, que le pragmatisme de Dewey vise une politique sans transcendance ${ }^{16}$. Ce que semble suggérer l'essai de 1939 c'est la possibilité de ne plus assimiler désormais la normativité politique au sacré et l'ordre politique à la violence, de ne plus vivre le rapportà l'autorité sous le mode de la dépersonnalisation pouvant conduire à la sujétion la plus complète. Pour le vérifier, il faut être attentif à l'écriture même de Dewey, et remarquer comment les motifs de la banalité et de l'utopie se conjuguent pour évoquer une liberté véritable ou, comme préfère le dire le philosophe, une morale (politique) véritable :

On trouvera peut-être que tout ce que j'ai dit est une série de banalités morales, de lieux communs. Alors, je répondrai

14. Dewey, J., La démocratie créatrice..., treizième alinéa.

15. Voir "Du primat de la démocratie sur la philosophie" in La sécularisation de la pensée, Paris, Seuil, 1988, p. 37 et ss.

16. Heineman, R., que nous avons déjà cité, écrit : "In light of Einstein's firm belief that God does not play dice with the world, one would be in respectable company in questionning Dewey's rejection of the metaphysical. As the essence of a creed by wich individuals must give meaning to their lives, Dewey's system is spartan and even depressing. (...) But history seems to indicate that individuals require an attachement to something more ethereal than satisfactory consequences of a material sort, and this suggests that there may exist paradigms of meaning more appropriate for human society than Dewey's instrumentalism"., op. cit., p. 125. 
que c'est justement pour cela que je l'ai dit. Se départir de l'habitude de considérer la démocratie comme quelque chose d'institutionnel et d'extérieurà soi, acquérir l'habitude de la traiter comme un mode de vie personnel, c'est comprendre que la démocratie est un idéal moral et que, dans la mesure où elle devient un fait, elle est un fait moral. C'est se rendre compte que la démocratie est une réalité uniquement si elle est un lieu de vie commun" ${ }^{17}$.

Au premier degré de l'exposé, la banalité que Dewey prête à son propos concerne le rappel des considérations aussi vieilles que l'idée de démocratie qu'offre son texte : quoi de plus conventionnel à cet égard que l'invitation à la participation et à la moralité? La sollicitation de l'engagement, en tant que platitude moralisante, contraste tout en faisant système avec l'utopie, avec la croyance au consensus politique proclamée par Dewey sur la base d'une véritable "foi» en la capacité de chacun d'être pleinement citoyen. Mais il faut voir comment le banal réinscrit l'idée de "communication" si importante pour Dewey : car si la démocratie fait de l'«expérience» la seule autorité, c'est que cette expérience réclame la mise en œuvre d'une communauté de parole sans laquelle nulle coopération n'est possible. Si le «banal», c'est l'autorité même du propos du philosophe, c'est bien parce qu'à travers sa parole se cherchent des "lieux communs" de vie, l'utopie d'une parole communautaire offrant à la paix une chance véritable. Dewey a d'autant plus de raisons d'insister sur cette idée de paix qu'il sait, au moment où il écrit, cette perspective grandement menacée.

Le philosophe dont la carrière connut un tel essor pendant ces années où il enseigna à Columbia, fut aussi un intellectuel dont l'activité le conduisit bien au-delà de son pays. Ses voyages au Japon et en Chine (1919-1921), en Turquie (en 1924, à l'invitation de Mustapha Kemal lui-même), en Russie

17. Dewey, J., La démocratie créatrice..., douzième alinéa. Je dois aux remarques de la traductrice de l'essai de Dewey, Sylvie Chaput, la considération des motifs de la banalité et de l'utople dont je tente icl une interprétation : je veux l'en remercier. 
(1928) et les textes qu'il fit paraître à l'occasion de ces déplacements confirmèrent sa réputation de chercheur, de théoricien de l'éducation et de défenseur de la démocratie. A ces brèves indications il fautajouter que le philosophe séjournera aussi à l'étranger bien après avoir pris sa retraite de la vie académique. Ce sera le cas en 1937 où il se rendra en Afrique du Sud, ainsi qu'en 1926 et en 1937 où il se déplacera au Mexique. Or, il importe d'évoquer de manière plus précise ce dernier voyage si l'on veut saisir quelques uns des événements qui marquèrent la réflexion consignée par le penseur dans son essai de 1939 sur La démocratie créatrice... : l'enjeu concerne, en effet, le phénomène du totalitarisme.

II ne saurait être question de retracer ici l'ensemble des positions adoptées par Dewey dans toute une partie de son œuvre à propos de la révolution de 1917 qui donna naissance à l'Union Soviétique, à propos du communisme en général ou du marxisme. On peut rappeler que le penseur avait signalé publiquement son intérêt pour l'ensemble des initiatives prises en U.R.S.S. au plan de l'éducation lors de son retour de voyage dans ce pays en 1928 et que, par la suite, il avait non seulement pris ses distances avec l'expérience soviétique, mais il avait combattu le programme politique et idéologique avancé par le socialisme tel qu'il pouvait en observer la mise en place dans divers pays. Les démêlés de Dewey avec les communistes américains et leurs sympathisants furent nombreux. Ceux causés par ses interventions à propos des purges staliniennes de la fin des années 30 s'ajoutèrent à d'autres soulevés par les réserves sérieuses du penseur quant à l'attitude de plusieurs intellectuels de gauche face à la guerre d'Espagne, au Front Populaire et à l'alliance avec les communistes dans la lutte antifasciste, etc.

C'est à l'occasion des fameux procès de Moscou que Dewey accepta de présider une commission d'enquête chargée d'étudier les accusations portées contre Trotsky par le régime de Staline. Bientôt connu sous le nom de Commission Dewey, ce regroupement fut mis sur pied en mars 1937 à l'initiative du Comité américain de défense de Léon Trotsky. Les commissaires 
qui accompagnèrent Dewey à Mexico en avril 1937 pour recueillir le témoignage de Trotsky et de son fils incluaient des trotskistes américains bien connus comme George Novack (qui agira comme secrétaire de la commission) et des intellectuels anti-staliniens comme James Burnham, V.F. Carlverton, John Dos Passos, Max Eastman, James T. Farrel, Sidney Hook, Horace M. Kallen, DwightMacdonald, Mary MacCarthy, Reinhold Niebuhr, Herbert Solow, Norman Thomas et Edmund Wilson. En décembre 1937, les commissaires faisaient connaître leur rapport dans lequel ils concluaient que Trotsky et son fils étaient innocents des crimes d'assassinat et de trahison dont les accusait Staline; du même coup, ils dénonçaient les procès de Moscou les dépeignant comme une pure et simple moquerie et une injustice flagrante. L'essai produit par Dewey en 1939 sur La démocratie créatrice...sera nettement marqué par la réflexion à laquelle le conviera son engagement dans les travaux de cette commission.

À ces événements des années 1937-1938 s'ajoutent bien d'autres péripéties politiques qu'on ne saurait présenter en détail. II suffira de dire que les prises de position de Dewey contre I'U.R.S.S. vont se multipler et le ton employé par le penseur, se durcir. Au retour de Mexico, et dans les années qui suivront, Dewey soulignera avec encore plus de vigueur l'importance de protéger la vie démocratique en s'assurant que les moyens pris pour l'élaboration et la mise en œuvre des décisions politiques soient évidemment eux-mêmes démocratiques.

Jusqu'à sa mort en 1952, l'engagement social et politique de Dewey, son combat pour l'idée de démocratie s'intensifiera l'invitant à associer son nom à diverses initiatives : elles touchentà l'organisation syndicale des enseignants, à la tentative pour mettre sur pied un troisième parti politique aux États-Unis, à l'appui aux socialistes de Norman Thomas aux élections de 1932, 1936 et 1940 comme à l'appui donné à Rosevelt aux élections présidentielles de 1944. On ne compte ici ni les articles parus dans divers périodiques et quotidiens, ni les échanges nombreux du penseur avec d'autres chercheurs et 
diverses personnalités de la société américaine et dont l'écho sera public ${ }^{18}$.

L'œuvre de Dewey est immense : le terme vaut autant pour son rayonnement philosophique que pour son ampleur. L'édition de ses œuvres par la Southern Illinois University Press (à Carbondale, Illinois) est en cours depuis la fin des années 60 et se poursuit. Au moins trente sept volumes compléteront l'ensemble de l'édition qui paraît en trois parties : The Early Works, 1882-1898, The Middle Works, 1898-1924 et The Later Works, 1925-1953 ${ }^{19}$. On n'aurait pas tort d'avancer que parmi l'ensemble des textes que réunit cette œuvre, le petit essai de 1939 que nous présentons ne peut rivaliser avec ces livres souvent cités que sont The Public and its Problems, Individualism, Old and New, ou Freedom and Culture. Et pourtant, La démocratie créatrice... a inspiré l'article de Bernstein que nous avons signalé; il informe à sa manière tout le chapitre que Cornel West consacre à Dewey dans le livre de ce commentateur que nous avons cité; et on en retrouve la trace active comme la mention explicite dans le "Commentaire" de Steven Rockefeller à la conférence inaugurale au "Centre pour les valeurs humaines", à l'université Princeton, prononcée par Charles Taylor et dont celui-ci a tiré l'essai «La politique de la reconnaissance" paru tout récemment dans Multiculturalisme

18. Quant à l'œuvre des dernières années, voici ce qu'avec le plus de raccourci possible on peut en évoquer à l'aide de ces lignes de Gérard Deledalle quil tiennent compte toutefois d'une période de temps un peu plus large que celle de la retraite de Dewey en 1930 : "Les écrits de philosophie politique et sociale les plus importants de Dewey sont les sulvants : German Philosophy and Politics (1915), The Public and its Problems (1927), Characters and Events (1930), deux volumes d'articles de lecture facile, Individualism, old and new (1930), A Common Faith (1934), Liberalism and Social Action (1935), Freedom and Culture (1939), Education Today (1940)", op. cit., p. 174.

19. La version de Creative Democracy - The Task Before Us dont nous offrons ici la traduction ne provient pas de cette édition. Voir plus loin la note de la traductrice précisant la provenance de cette version. Dans l'édition de la Southern Illinois University Press, on la retrouve toutefois dans le volume 14 des Later Works paru en 1988, aux p. 224-230. 
- Différence et démocratie ${ }^{20}$. On le voit: ce petit texte, œuvre de circonstance, resurgit à l'occasion pour rappeler l'essentiel de ce que Dewey avait engagé dans sa lutte et sa réflexion politiques.

L'intérêt pour cette lutte et cette réflexion se fait aujourd'hui grandissant : on en jugerait facilement par les considérations que leur consacrent des penseurs comme Richard Rorty ou Hilary Putnam ${ }^{21}$. Cen'est pas vraiment un hasard sile philosophe Cornel West a pu écrire :

Le combat de Dewey pour garder vivant le meilleur de la religion del'Amériquen'est pas simplement incisif et instructif : il est étonnant et inspirant. II est resté trop longtemps enfoui dans l'inconscient de l'Amérique, vénéré parcertains épigones chauvins, déprécié par certains spécialistes myopes et pourtant peu l'ont interrogé avec sérieux. II mérite un meilleur sort. En fait, je crois qu'on assistera bientôt à un nouvel essor de Dewey. J'espère tout simplement que ce retour s'accomplira avec un sens plus aigu de l'histoire, une mise en œuvre plus subtile et plus nuancée de l'esprit critique et un engagement accru dans le développement de la démocratie créatrice ${ }^{22}$.

Ces lignes annoncent moins une échéance qu'elles ne traduisent un espoir: et il est certes difficile de présumer du sort qui lui est réservé. L'optimisme impénitent de Dewey, sa réputation de penseur libéral et américain comme sa tentative pour donner forme à une véritable morale politique, risquent de faire obstacle à la lecture de son œuvre : après tout, en cette fin de siècle où s'alourdit le bilan des rêves politiques brisés, la tentation est grande de conclure à l'impossibilité d'une véritable

20. Taylor, C., Multiculturalisme - Différence et démocratie, avec des commentaires de Amy Gutmann - Steven C. Rockefeller - Michael Walzer - Susan Wolf, traduit de l'américain par Denis-Armand Canal, Paris, Aubier, 1994.

21. De Richard Rorty, on peut lire "Du primat de la démocratie sur la philosophie" in La sécularisation de la pensée, Paris, Seull, 1988; voir aussi ses Consequences of Pragmatism, University of Minnesota Press, Mineapolis, 1989. De Hilary Putnam on peut lire plus particulièrement "A Reconsideration of Deweyan Democracy" dans son Renewing Philosophy, Harvard University Press, Cambridge, Massachussetts, London, England, 1992.

22. West, C. The American Evasion of Philosophy..., p. 86 (nous traduisons). 
communauté éthique. À tout le moins, il semble acquis qu'il faudra encore pour longtemps faire ses choix moraux en fonction d'un monde où règnent toujours l'injustice et la guerre. Pour pacifier les rapports sociaux et même les moraliser, la pensée politique moderne avait fait appel à la démocratie, à la souveraineté populaire et au droit constitutionnel qui lui sont attachés : c'est à ces conditions que le citoyen pouvait espérer être protégé du mal et de l'arbitraire. Dewey ne demande pas que l'on abandonne cette perspective : il nous invite à la comprendre d'une façon nouvelle en cherchant à délimiter autrement la frontière entre l'éthique et la politique. C'est dire que le penseur exige que la démocratie soit plus qu'un rempart contre l'oppression : plus que jamais, comme Dewey le savait déjà, le succès de cette politique négative est compromis. La démocratie doit procurer à chacun une valeur positive, une justice palpable. Pour la définir, il ne suffira pas d'introduire une finalité politique en extériorité; il faudra proposer à chacun un engagement possible et se nourrir de la créativité dont il est capable. C'est à réfléchir aux conditions de possibilité d'un tel engagement que nous convie Dewey en disant de la "démocratie créatrice" qu'elle est "la tâche qui nous attend».

Francois Leroux, Département de philosophie Collège Edouard-Montpetit 


\section{BIBLIOGRAPHIE COMPLÉMENTAIRE}

\section{Ouvrages de John Dewey parus en traduction française.}

L'école et l'enfant, traduction de L. S. Pidoux, Neuchâtel, Delachaux et Niestlé, 1913 (recueil de quatre textes allant de 1896 à 1902).

Mon Credo pédagogique, traduction de Ou Tsuin-Chen, Paris, Vrin, 1931, réed. 1958 (My Pedagogic Creed, 1898).

L'école et la société, traduction partielle dans L'éducation, juin 1909 et décembre 1912 (The School and Society, 1900).

Comment nous pensons, traduction de O. Decroly, Paris, Flammarion, 1925 (traduction de la première édition de How We Think, 1910; Dewey en a publié une édition augmentée en 1933).

Les écoles de demain, traduction de R. Duthil, Paris, Flammarion, 1931 (Schools of Tomorrow, 1915).

Démocratie et éducation, présentation et traduction de Gérard Deledalle, Paris, Armand Colin, Bibliothèque européenne des sciences de l'éducation, 1990, (1975). Paru également à l'Age d'homme en 1983 (Democracy and Education - An Introduction to the Philosophy of Education, 1916).

Expérience etéducation, avecune présentation de la pédagogie de Dewey par M. A. Carroi, Editions Bourrelier et Cie, 1947; nouvelle édition Armand Colin, 1968 (Experience and Education, 1938).

Logique : La théorie de l'enquête, présentation et traduction de Gérard Deledalle, Paris P.U.F., 1967 (Logic: The Theory of Inquiry, 1938).

Liberté et culture, traduction de Pierre Messiaen, Paris, Aubier, 1955 (Freedom and Culture, 1939). 


\section{Ouvrages sur John Dewey et la philosophie américaine.}

Deledalle, G., La pédagogie de John Dewey, Paris, Éditions du Scarabée, coll. «Faits et doctrines pédagogiques», 1965.

Deledalle, G., L'idée d'expérience dans la philosophie de John Dewey, Paris, P.U.F., 1967.

Hook, S., John Dewey, An Intellectual Portrait, Greenwood Press, Publishers, Westport, Connecticut, 1939.

Horwitz, R., "John Dewey" in History of Political Philosophy, 3e ed., Straus, L. \& Cropsey, J.,ed., The Chicago University Press, Chicago and London, 1987.

MacKinnon, B., Ed., American Philosophy - A Historical Anthology, State University of New York Press, Albany, 1985 ( cf le chapitre 8 et une partie du chapitre 17 consacrés à Dewey).

Marcuse, L., La philosophie américaine, Paris, Gallimard, coll. «ldées», 1967.

Vacher, L.-M., L'empire du moderne-Actualité de la philosophie américaine, Montréal, Les Herbes rouges, 1990.

West, C., «La politique du néo-pragmatisme américain» in Rajchman, J., \& West, C. (sous la direction de), La pensée américaine contemporaine, traduit de l'américain par Andrée Lyotard-May, Paris, P.U.F., coll. «Philosophie d'aujourd'hui», 1991. 